\title{
Interaction écoulement/mouvement par un solveur Navier-Stokes avec adaptation locale de maillage; application à l'étude du slamming
}

\author{
Alban Leroyer $^{1, a}$, Alexander Hay $^{2}$ et Michel Visonnead ${ }^{1}$ \\ 1 École Centrale de Nantes, Laboratoire de Mécanique des Fluides UMR CNRS 6598, Équipe Modélisation Numérique, \\ BP 92101, 1 rue de la Noë, 44321 Nantes Cedex 3, France \\ 2 École Polytechnique de Montréal, Département de Génie Mécanique, C.P. 6079, Succursale Centre-ville, Montréal, Québec, \\ Canada, H3C3A7
}

Reçu le $1^{\text {er }}$ septembre 2006, accepté le 23 janvier 2007

Résumé - Cet article présente la simulation d'écoulements autour de corps en mouvement basée sur un solveur Navier-Stokes utilisant des techniques d'adaptation locale de maillage. Après une description des méthodes utilisées, une application à l'étude d'impacts sera présentée. On mettra en évidence l'intérêt de l'adaptation locale de maillage dans ce type de simulation, notamment pour la capture précise des pics de pression engendrés lors des impacts.

Mots clés : Solveur Navier-Stokes / interaction fluide-structure / surface libre / adaptation automatique de maillage / slamming

\begin{abstract}
Flow/motion interaction using a Navier-Stokes solver with automatic local grid refinement; application to slamming. This paper deals with Navier-Stokes simulations of flows around moving bodies coupled with an adaptive mesh refinement strategy. Firstly, a description of the considered methods is given. Then, an application involving hydrodynamic impacts is thoroughly presented and studied. The usefulness of the adaptive procedure is clearly demonstrated, especially regarding the accurate computation of pressure peaks arising during impacts.
\end{abstract}

Key words: Navier-Stokes solver / fluid-structure interaction / free surface / h-adaptive methods / slamming

\section{Introduction}

L'augmentation des capacités de calcul et de stockage permet aujourd'hui d'envisager des simulations plus réalistes mais aussi plus complexes. L'étude d'écoulements réels autour de corps en mouvement fait partie de ces nouvelles applications qui deviennent désormais abordables en terme de temps de calculs. Les applications industrielles de ce type de simulations sont nombreuses, notamment dans le domaine de l'hydrodynamique : tenue à la mer, étude du comportement de tanker, phénomène de slamming, ...

Après une description succincte des principales caractéristiques du code de calcul ISIS-CFD développé au sein de l'Équipe Modélisation Numérique du Laboratoire

\footnotetext{
a Auteur correspondant : Alban.Leroyer@ec-nantes.fr
}

de Mécanique des Fluides de l'ECN, des méthodes liées aux mouvements de corps (remaillage, couplage entre les équations de Navier-Stokes et le Principe Fondamental de la Dynamique noté par la suite PFD) ainsi que des stratégies d'adaptation locale de maillage, l'ensemble des techniques présentées sera appliqué à l'étude d'impacts d'un corps prismatique. Dans ce cadre, on montrera l'intérêt de l'association mouvement de corps/adaptation de maillages pour envisager à l'avenir de nouvelles applications.

\section{Présentation du code de calcul}

Le code ISIS-CFD est basé sur la résolution des équations de Navier-Stokes en moyenne de Reynolds (RANSE) sous une forme fortement conservative. Il 


\section{Nomenclature}

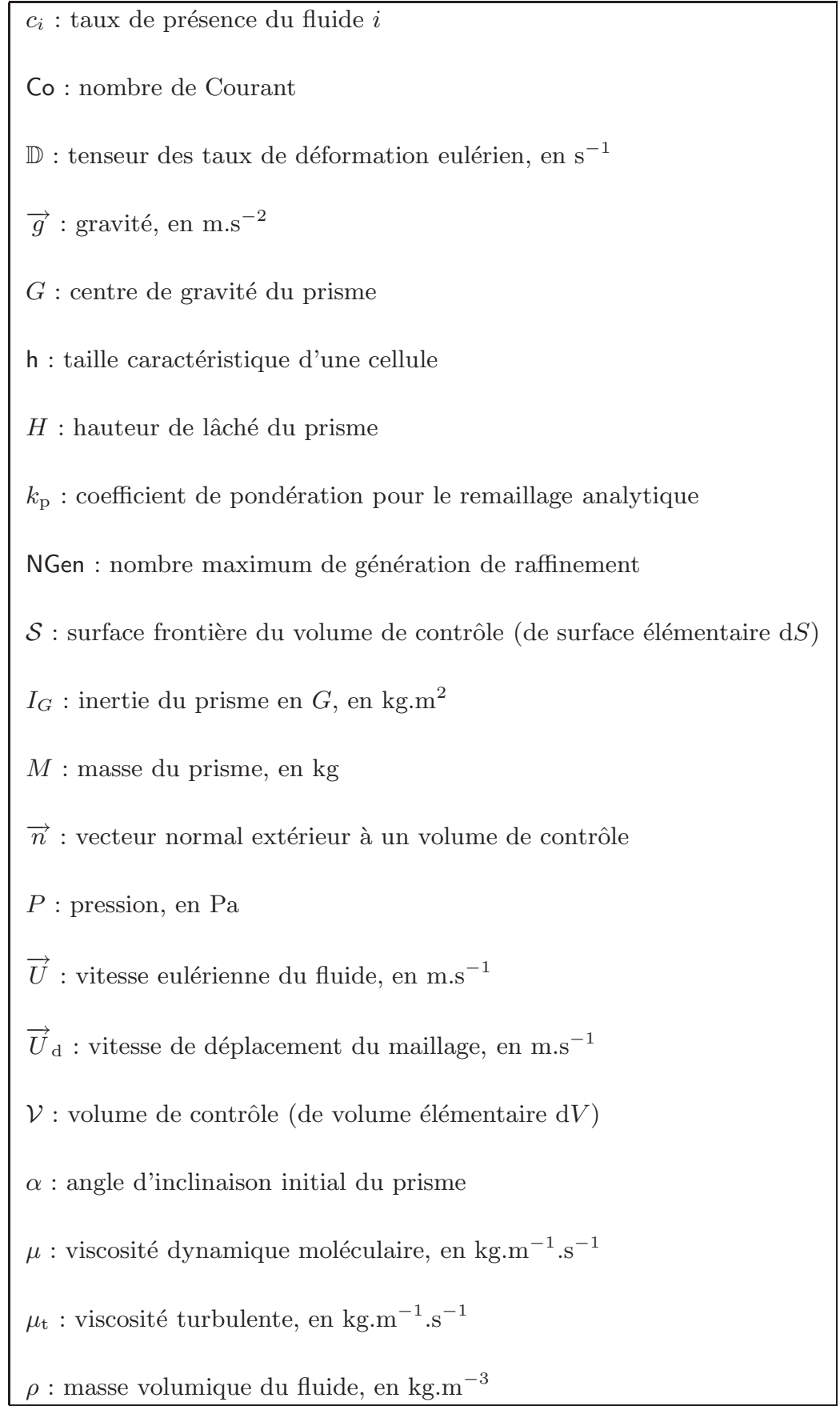

repose sur une méthode de volumes finis généralisée à des maillages non-structurés composés de volumes polyédriques quelconques.

Le champ de vitesse est obtenu à partir des équations de conservation de quantité de mouvement et la pression est déterminée selon la contrainte d'incompressibilité transformée en équation de pression. Le couplage vitessepression est assuré par un algorithme de type SIMPLE [1]. L'ensemble des variables est stocké aux centres des volumes de contrôle. Les intégrales de volume et de surface sont évaluées par des approximations précises au second ordre. Les calculs multiprocesseurs sont gérés par MPI.

Il est aussi possible de simuler des écoulements avec surface libre [2]. Ils sont gérés par une méthode de capture d'interface. L'ensemble des deux phases est vu comme un fluide unique dont les propriétés varient avec un taux de présence. Celui-ci est traité comme un lagrangien emporté par le fluide global. Sa discrétisation nécessite des schémas appropriés pour éviter une diffusion trop importante de l'interface. 
La discrétisation temporelle pour les calculs instationnaires est une méthode implicite du deuxième ordre décentrée à deux pas de temps. À chaque pas de temps, une boucle interne (appelée «boucle non-linéaire ») associée à une linéarisation de Picard est effectuée pour résoudre les non-linéarités. Le passage à l'itération temporelle suivante est opéré lorsque le résidu des équations a été réduit d'un certain gain (typiquement 3 ordres).

Les équations résolues sont présentées ci-dessous $(\delta / \delta t$ désigne la dérivée par rapport au temps selon le champ de vitesse $\vec{U}_{\mathrm{d}}$, c'est-à-dire « en suivant le maillage dans son mouvement ») :

Propriétés globales du fluide :

$$
\sum_{i} c_{i}=1, \rho=c_{i} \rho_{i}, \mu=c_{i} \mu_{i}
$$

Loi de conservation géométrique :

$$
\frac{\delta}{\delta t} \int_{\mathcal{V}} \mathrm{d} V-\oint_{\mathcal{S}} \vec{U}_{\mathrm{d}} \cdot \vec{n} \mathrm{~d} S=0
$$

Fonction de présence :

$$
\frac{\delta}{\delta t} \int_{\mathcal{V}} c_{i} \mathrm{~d} V+\oint_{\mathcal{S}} c_{i}\left(\vec{U}-\vec{U}_{\mathrm{d}}\right) \cdot \vec{n} \mathrm{~d} S=0
$$

Conservation de la quantité de mouvement :

$$
\begin{aligned}
\frac{\delta}{\delta t} \int_{\mathcal{V}} \rho \vec{U} & \mathrm{~d} V+\oint_{\mathcal{S}} \rho \vec{U}\left(\vec{U}-\vec{U}_{\mathrm{d}}\right) \cdot \vec{n} \mathrm{~d} S \\
& =\int_{\mathcal{V}}(-\vec{\nabla} P+\rho \vec{g}) \mathrm{d} V+\oint_{\mathcal{S}} 2\left(\mu+\mu_{t}\right) \mathbb{D} \cdot \vec{n} \mathrm{~d} S
\end{aligned}
$$

Conservation de la masse :

$\oint_{\mathcal{S}} \vec{U} \cdot \vec{n} \mathrm{~d} S=0 \rightarrow$ équation de pression.

\section{Gestion des mouvements de corps}

\subsection{Résolution du PFD et couplage}

ISIS-CFD a été étendu pour simuler les mouvements 3D d'un nombre quelconque de corps indéformables (solides) mais aussi déformables à déformation imposée (la forme du corps est alors imposée au cours du temps). Les degrés de liberté pour chaque corps peuvent être soit résolus soit imposés en donnant explicitement la loi d'évolution du paramètre dans le repère physique.

Dans l'application bidimensionnelle présentée ici, la résolution du PFD ne pose pas de problème particulier. On se reportera à [3] et à [4] pour plus de détails sur la résolution générale.

Concernant le couplage avec la résolution de l'écoulement, celui-ci existe uniquement lorsque le mouvement (ou une partie du mouvement) est résolu par application du PFD. La cinématique des corps à l'instant de calcul est alors liée à l'écoulement à ce même instant

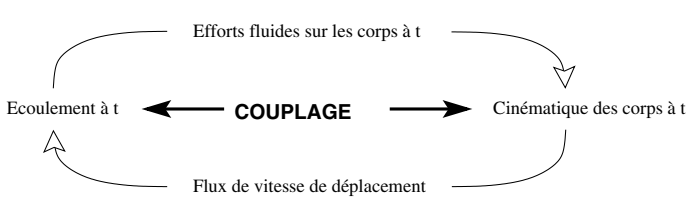

Fig. 1. Schématisation du couplage écoulement-mouvement.

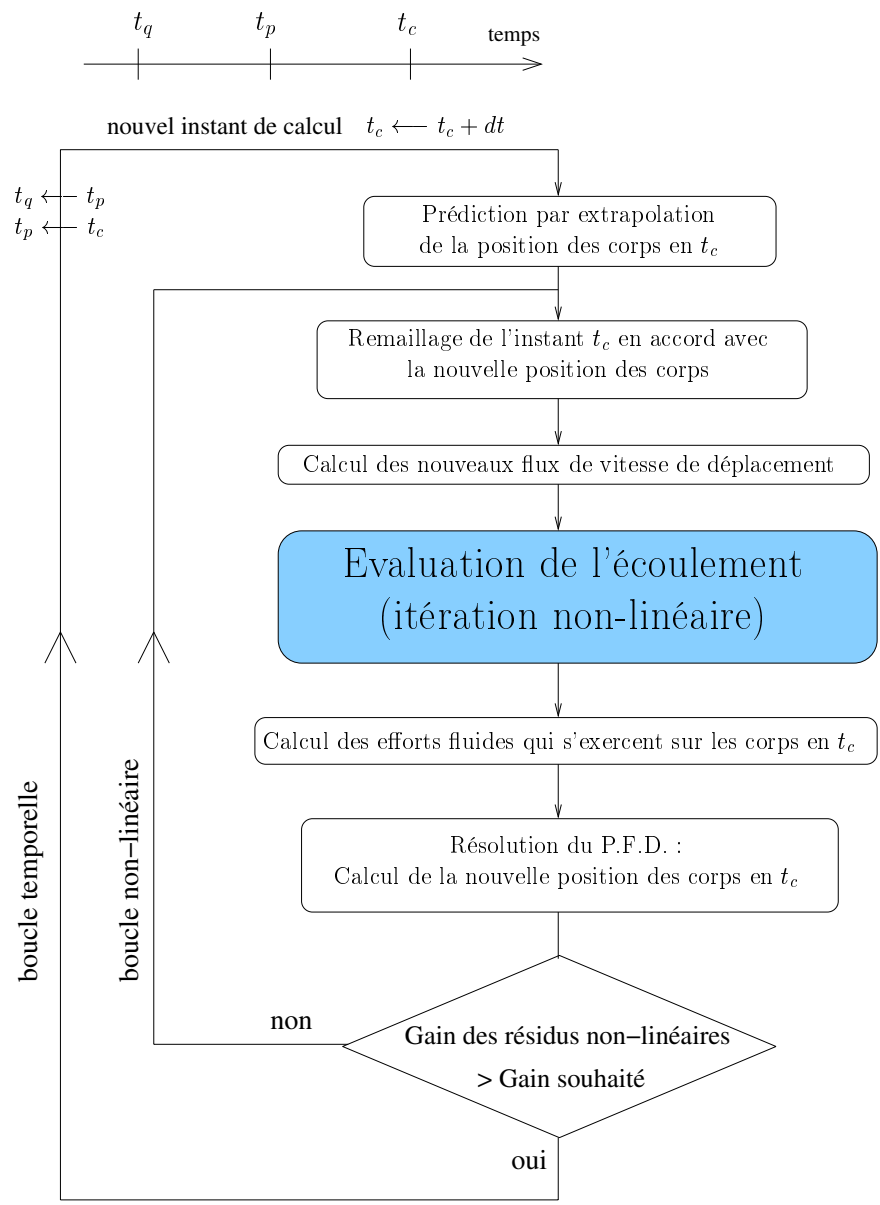

Fig. 2. Algorithme du couplage non-linéaire.

par les efforts fluides qui s'exercent sur eux. En retour, le mouvement des corps influence l'écoulement par l'intermédiaire des flux de vitesse de déplacement (Fig. 1).

Pour obtenir un couplage stable, il s'est avéré obligatoire de remettre à jour l'interaction écoulementmouvement non plus à l'issue de la boucle temporelle mais dans la boucle non-linéaire. Les efforts sont ainsi recalculés à chaque itération non-linéaire et servent à réajuster la position des corps pour l'itération non-linéaire suivante par l'intermédiaire du PFD. Après remaillage, les nouveaux flux de vitesse de déplacement engendrés par cette nouvelle cinématique sont réévalués. On peut noter ici l'intérêt de disposer de procédures de remaillage analytique qui permettent de ne pas pénaliser le temps de calcul. L'interaction écoulement-mouvement est ainsi complètement actualisée à chaque itération non-linéaire. Cela se traduit par l'algorithme présenté sur la figure 2. 


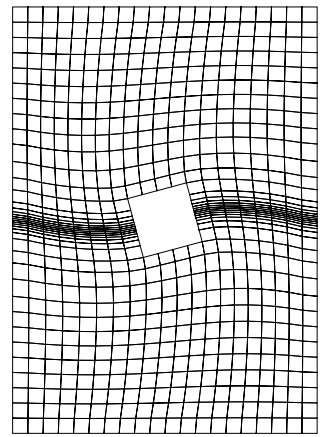

(a) $15^{\circ}$

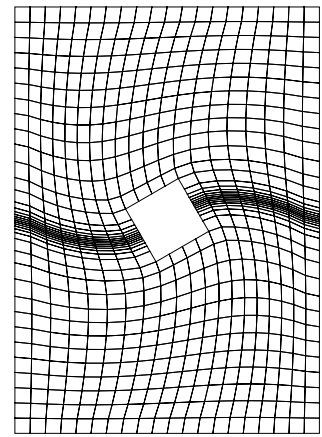

(b) $30^{\circ}$

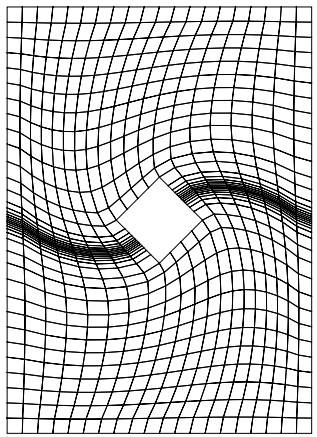

(c) $45^{\circ}$

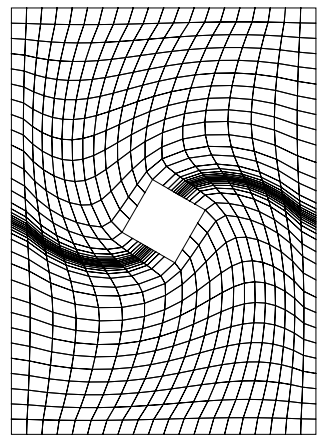

(d) $60^{\circ}$

Fig. 3. Remaillage par pondération pour différents angles de rotation.

\subsection{Remaillage}

ISIS-CFD, comme la plupart des solveurs RANSE utilise des maillages qui s'appuient sur la surface des corps («body-fitted grid»). L'emploi de maillages exclusivement cartésiens « non body-fitted » dans lequel les corps sont immergés (« immersed boundary» avec éventuellement un raffinement local près des corps) reste en effet pour le moment inadapté pour représenter précisément les couches limites turbulentes près des corps. ISIS-CFD utilise un seul maillage, excluant les méthodes à recouvrement de maillages de type « chimère ». Cela permet d'éviter les problèmes d'interpolation entre les différents maillages. En contrepartie, il est nécessaire de gérer le déplacement au cours du temps des nœuds du maillage représentant chaque corps dans le repère physique. Pour conserver un maillage adéquat, différentes méthodologies ont été développées. Elles peuvent être utilisées de manière hybride suivant les degrés de liberté : remaillage par analogie à une pseudo-structure (résolution d'un problème d'élasticité linéaire avec module d'Young variable ou analogie du domaine fluide à un assemblage de ressorts), repositionnement en bloc et remaillage par pondération analytique (pour les mouvements rigides et de type poutre). Les techniques basées sur une pseudo-structure permettent des déformations de forme quelconque assez importantes mais nécessitent la résolution d'un système linéaire. Le repositionnement en bloc n'est pas à proprement parler une technique de remaillage puisque dans ce cas le maillage suit exactement le mouvement du corps sans se déformer. La qualité du maillage est donc conservée, mais cela n'est applicable que pour des calculs mettant en jeu un corps unique en milieu infini. À noter que dans ce cas, l'évolution temporelle de la transformation rigidifiante (translation+rotation) appliquée aux points du maillage est décrite par un torseur cinématique dont les caractéristiques servent au calcul (exact) des flux de vitesse de déplacement des faces du maillage par rapport au repère physique.

Le remaillage par pondération analytique peut être vu comme une extension du repositionnement en bloc, mis à part que l'on pondère le mouvement du corps suivant la proximité du corps par un coefficient $k_{\mathrm{p}}$ calculé initialement. Celui-ci vaut 1 pour les nœuds du corps considéré,
0 pour ceux des autres frontières du domaine de calcul. Il est obtenu en début de simulation par résolution d'un laplacien sur la configuration de référence. Un exemple pour un carré effectuant une rotation de $60^{\circ}$ est donné sur la figure 3. Le temps CPU quasiment négligeable permet à cette technique de se substituer avantageusement aux méthodes pseudo-structure, notamment pour des amplitudes de déformation raisonnables. Une extension de cette technique a été réalisée pour les corps déformables de type poutre (voir [3]). Pour plus de détails sur ces stratégies de remaillage, on se reportera à [4].

\section{Techniques d'adaptation locale de maillage}

Une méthode d'adaptation locale de maillage nonstructuré ( $h$-adaptation) a été intégrée dans le code ISISCFD (voir [5]). D'une manière générale, l'objectif de celleci est d'adapter les grilles de calcul à la solution numérique recherchée au cours d'un processus automatique en minimisant les coûts de calcul et l'effort humain.

Pour les simulations instationnaires qui nous concernent plus précisément ici, ces techniques ont été mises à profit dans des applications multi-phases, afin obtenir une description précise de la surface libre [6].

\subsection{Structure de données}

Pour que le processus adaptatif soit flexible, rapide et facile à mettre en œuvre, une structure de données adéquate est nécessaire. Ainsi, la procédure développée dans le cadre de cette étude est basée sur la notion de parenté entre les générations successives d'éléments des grilles de calcul. Des connectivités de parenté sont mises en place pour les volumes et les faces des maillages. Elles sont présentées ici uniquement pour les éléments de type volume mais des notions similaires existent également pour les éléments de type face. Les relations de parenté introduites entre les cellules des maillages empruntent un vocabulaire naturel de famille, père, fils et frère comme illustré sur la figure 4. Le maillage initial est constitué des éléments de générations 0 et une première étape de raffinement crée des éléments de génération 1. 


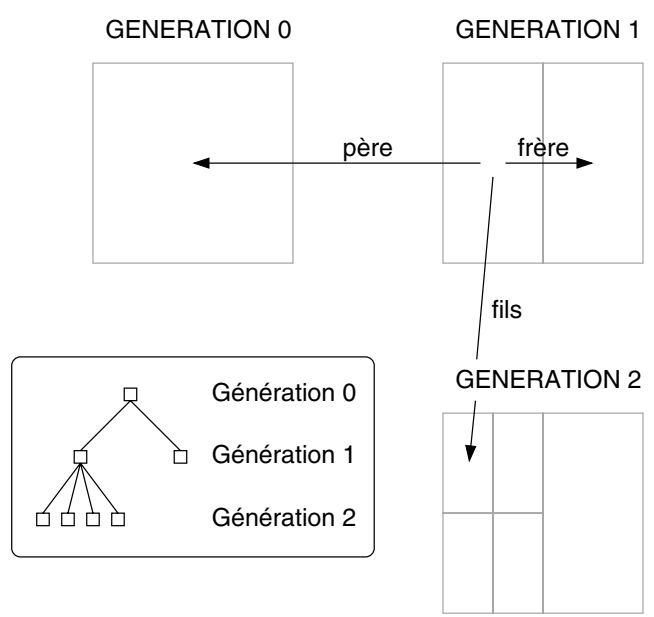

Fig. 4. Relations de parenté entre les différentes générations d'éléments.

Un volume de contrôle raffiné devient père et il est divisé en plusieurs volumes fils qui partagent entre eux une relation de frère. En outre, un père et ses fils forment une famille. Une étape d'adaptation supplémentaire aboutit aux éléments de génération 2 et ainsi de suite. Une telle structure de données ne réduit en rien la généralité de la procédure adaptative puisqu'elle ne tient pas compte de la façon dont les éléments des grilles sont modifiés. Par ailleurs, il peut exister des éléments de générations négatives créés lors du déraffinement par agglomération des grilles initiales [5]. Il est important de noter que les éléments des générations successives sont toujours gardés en mémoire (ils ne sont détruits que par déraffinement). Cependant le coût supplémentaire en terme de mémoire est très réduit puisque l'adaptation est toujours très localisée.

Une telle structure de données permet de résoudre plusieurs problèmes à la fois. Tout d'abord, le déraffinement d'une famille (c'est-à-dire d'une cellule précédemment raffinée) devient immédiat puisque cette opération consiste alors simplement à réactiver le père et détruire les fils. Ainsi, le processus de raffinement/déraffinement est très dynamique et rapide. De plus, il permet de revenir au maillage initial dans les zones où l'adaptation réalisée n'est plus nécessaire. C'est notamment le cas lors des simulations instationnaires.

\subsection{Modification des éléments}

Au cours du processus adaptatif, chaque volume de contrôle devant être raffiné est divisé en plusieurs volumes plus petits mais de même topologie. Ainsi, la topologie des éléments en tout point du maillage est toujours la même et seules leurs tailles sont adaptées. Dans le cas bidimensionnel, comme illustré sur la figure 5 , le raffinement peut être effectué de manière isotrope ou directionnelle permettant de tenir compte de certaines propriétés particulières des écoulements considérés.

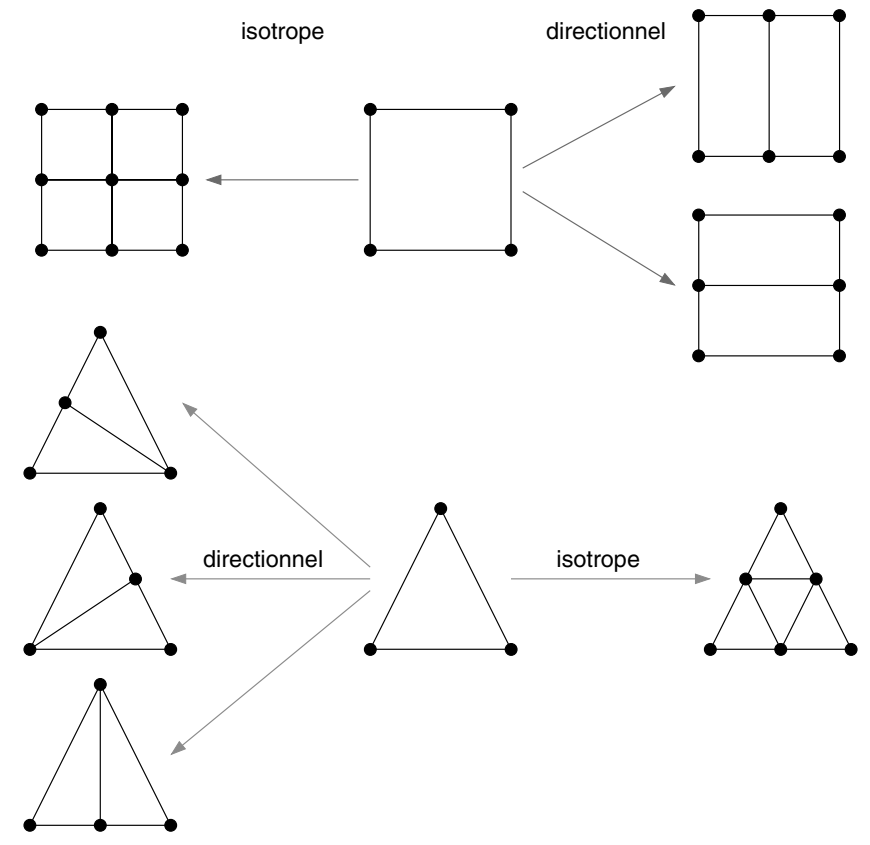

Fig. 5. Raffinement d'un volume de contrôle 2D.

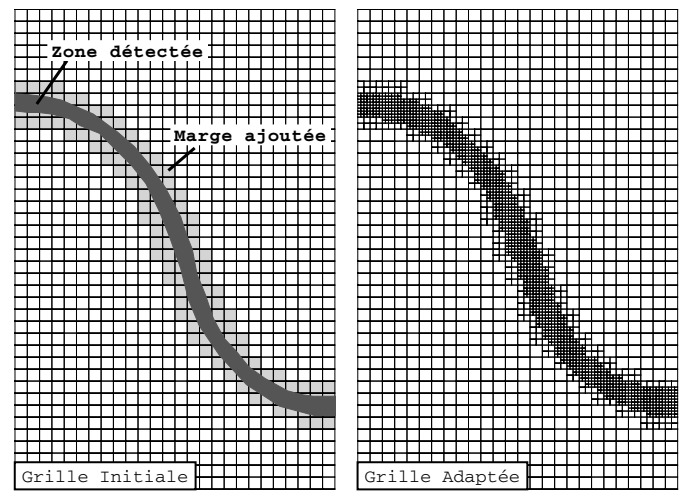

Fig. 6. Marge ajoutée autour des zones sélectionnées.

Une fois modifiées, les grilles de calculs ne nécessitent aucun traitement particulier au sein du solveur NavierStokes. En effet, un voisin non raffiné d'une cellule raffinée présente un nœud libre (hanging-node) qui est traité naturellement par la méthode volumes finis basée sur les faces : une face possédant un hanging-node est simplement considérée comme plusieurs faces plus petites.

\subsection{Description de la procédure}

Dans le cadre de cette étude, le but de la procédure adaptative est de maintenir une taille caractéristique de maille assez petite au voisinage de la surface libre, afin de limiter sa diffusion numérique. Un indicateur explicite est donc nécessaire de manière à identifier la position de cette interface dans le domaine de calcul. Un tel indicateur est facile à mettre en place en se basant sur la fraction volumique $c_{i}$. Ainsi, on considère que l'interface se situe dans les cellules pour lesquelles $c_{i}$ est compris entre 0,05 


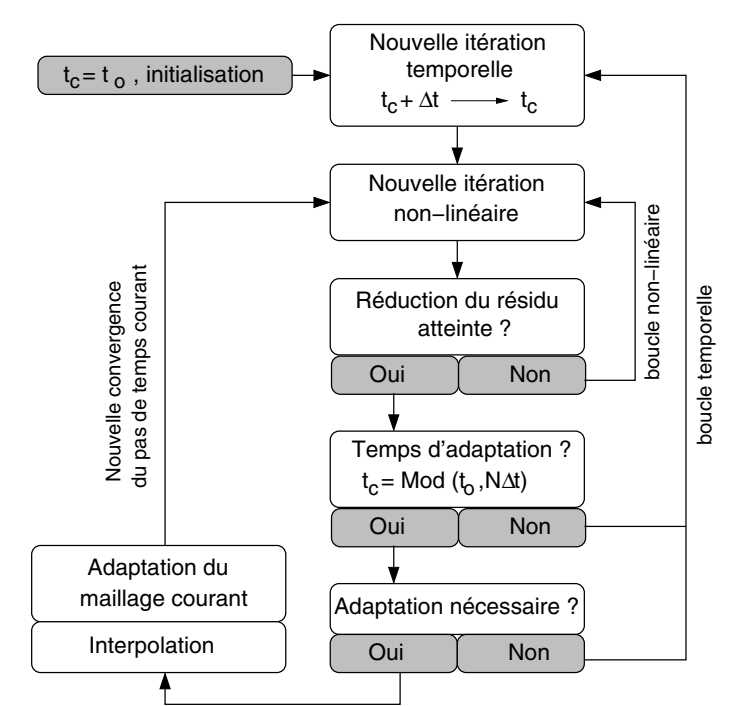

Fig. 7. Schéma de la procédure : cas instationnaire.

Tableau 1. Paramètres des différentes simulations réalisées.

\begin{tabular}{ccccc}
\hline Nom & SymLight SymMedium & AsymLight AsymMedium \\
\hline Hauteur $H$ & $0,61 \mathrm{~m}$ & $0,61 \mathrm{~m}$ & $0,61 \mathrm{~m}$ & $0,61 \mathrm{~m}$ \\
\hline Angle $\alpha$ & $0^{\circ}$ & $0^{\circ}$ & $5^{\circ}$ & $5^{\circ}$ \\
\hline Masse $M$ & $122 \mathrm{~kg}$ & $291 \mathrm{~kg}$ & $124 \mathrm{~kg}$ & $293 \mathrm{~kg}$ \\
\hline Distance $C G$ & - & - & $0,216 \mathrm{~m}$ & $0,165 \mathrm{~m}$ \\
\hline Inertie $I_{\mathrm{G}}$ & - & - & $8,85 \mathrm{~kg} \cdot \mathrm{m}^{2}$ & $10,95 \mathrm{~kg} \cdot \mathrm{m}^{2}$ \\
\hline
\end{tabular}

et 0,95 . Une fois ces cellules identifiées, une marge de sécurité est ajoutée autour comme indiqué sur la figure 6 . Celle-ci permet d'assurer que la surface libre ne traverse jamais les frontières des zones raffinées.

La figure 7 présente une synthèse de l'algorithme de la procédure adaptative pour des simulations instationnaires. Une étape d'adaptation peut se produire, si nécessaire, tous les $N$ pas de temps durant la boucle de résolution instationnaire du solveur Navier-Stokes. Ce paramètre est toujours choisi petit de manière à ce que le maillage puisse suivre les évolutions temporelles de la surface libre. Comme indiqué sur le schéma, si aucune adaptation significative n'est nécessaire, le calcul se poursuit comme lors de simulations classiques. Si le maillage est modifié, une procédure de restriction/prolongation permet d'interpoler la solution courante sur la grille nouvellement adaptée permettant d'assurer la continuité de la simulation. L'opérateur d'interpolation utilisé garantit la préservation des propriétés conservatives des variables traitées comme cela est requis par la méthode des volumes finis [5]. Cependant, la solution nouvellement interpolée n'a aucune raison de satisfaire l'opérateur de Navier-Stokes instationnaire à l'instant courant. Ainsi, il est nécessaire de réaliser une nouvelle convergence du pas de temps courant comme indiqué sur la figure 7 . Le coût supplémentaire en temps de calcul dû à ces pas de temps additionnels au sein de la boucle instationnaire fait partie intégrante du coût global de la méthode adaptative.

La taille caractéristique $\mathrm{h}$ des cellules dans le voisinage de la surface libre est contrôlée par le paramètre
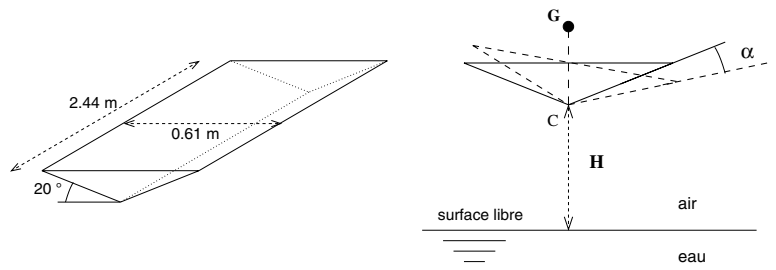

(a) Géométrie du corps pris- (b) Paramètres expérimatique

mentaux

Fig. 8. Configuration expérimentale.

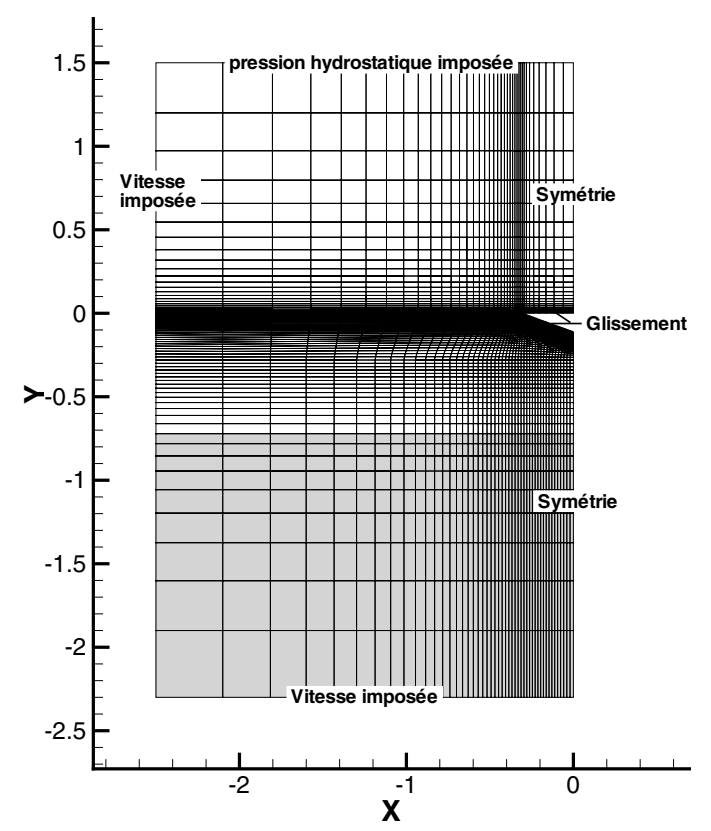

Fig. 9. Configuration numérique (cas symétrique).

NGen qui correspond au nombre maximum de générations autorisées sur les grilles de calcul. Ainsi, h peut au plus être réduit d'un facteur $2^{\text {NGen }}$ dans chaque direction. Plus NGen est choisi grand et plus la description de la surface libre sera précise.

\section{Application}

On s'intéresse ici au calcul de l'écoulement instationnaire autour d'un corps prismatique, lâché dans l'air et qui vient impacter la surface libre de l'eau. L'objectif sous-jacent à ce problème est l'étude des phénomènes de slamming. Le corps prismatique étudié est très allongé. Il est muni d'accéléromètres qui donnent accès à la cinématique du corps lors de l'impact sur la surface libre. Les paramètres de l'étude sont la hauteur $H$ de laquelle le corps est lâché, l'angle $\alpha$ d'inclinaison initiale et la masse $M$ du prisme (voir Fig. 8). Parmi toutes les expériences réalisées, notre étude s'est focalisée sur celles dont la hauteur de lâché $H$ est de $0,61 \mathrm{~m}$. Les différents cas étudiés sont rassemblés dans le tableau 1.

Les résultats des simulations (symétriques et asymétriques) vont être comparés aux données 


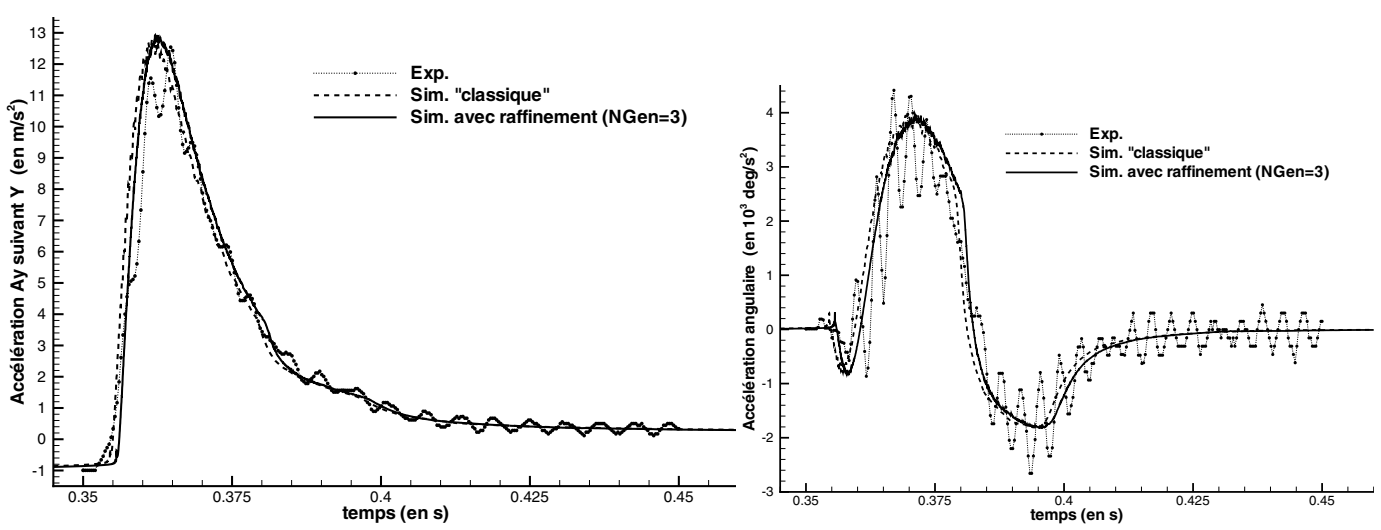

(a) Cas AsymLight : accélération suivant Y

(b) Cas AsymLight : accélération angulaire
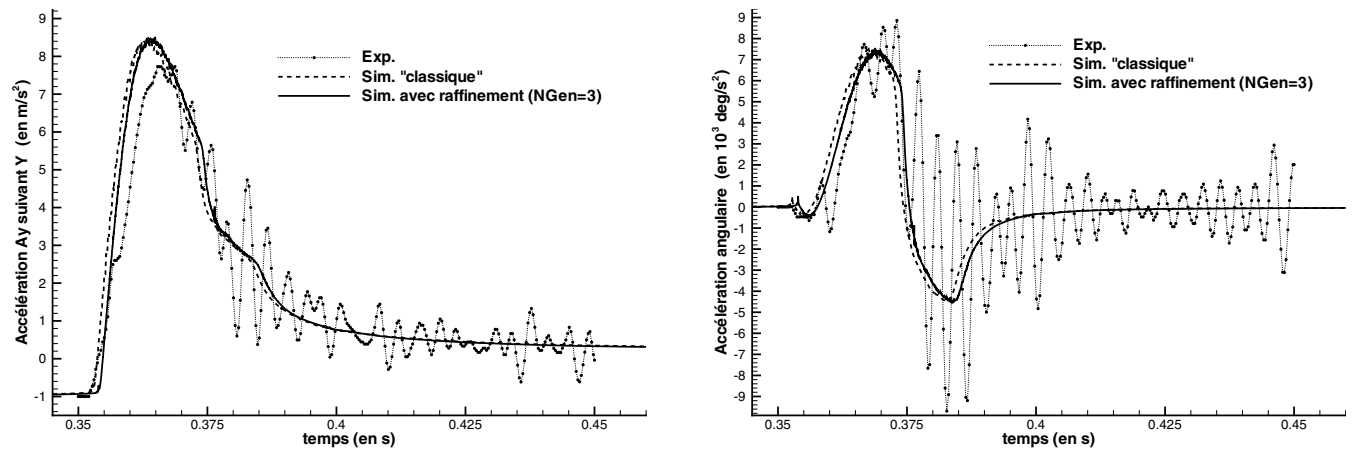

(c) Cas AsymMedium : accélération suivant Y

(d) Cas AsymMedium : accélération angulaire

Fig. 10. Comparaisons expériences-simulations.

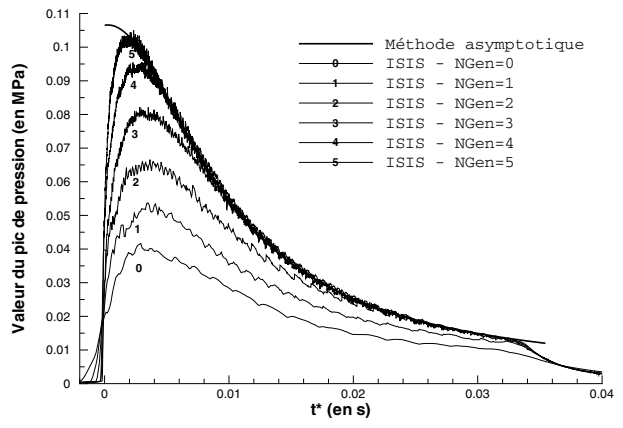

(a) Cas SymLight

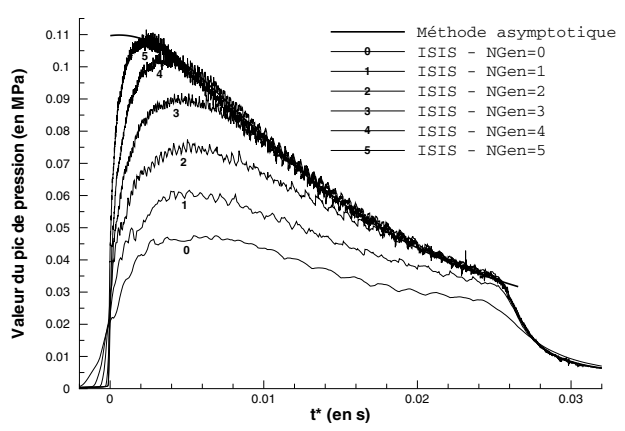

(b) Cas SymMedium

Fig. 11. Évolution temporelle de l'intensité du pic de pression : cas symétrique.

expérimentales disponibles dans [7]. Les cas symétriques vont faire l'objet d'une analyse plus précise sur la capture du pic de pression généré lors de l'impact, qui est une des principales caractéristiques d'étude des problèmes de slamming.

À partir d'une configuration de base de 3400 cellules pour le cas symétrique (voir Fig. 9), des simulations ont été réalisées pour chaque cas en autorisant un nombre croissant de niveaux de raffinement maximum NGen de 0 à 5. L'indicateur d'erreur qui identifie les cellules du maillage où se situe la surface libre est basé sur l'analyse de la fonction de présence. Une marge de sécurité est ensuite ajoutée autour des zones marquées. Durant les simulations, l'adaptation de maillage est proposée toutes les 10 itérations temporelles de manière à opérer le suivi de la surface libre sur la grille de calcul (voir Fig. 15). Pour limiter le nombre de points de calcul, le raffinement isotrope est utilisé uniquement dans une bande verticale proche du corps (pour $|x|<0,75 \mathrm{~m}$ ), le reste du domaine est affecté d'un raffinement directionnel. Cette opération ne dégrade pas la précision de la solution, car loin du corps, la surface libre (qui reste quasiment horizontale) n'a besoin d'être raffinée que dans sa direction orthogonale. Une zone de transition est constituée pour ne pas avoir des mailles voisines avec des rapports d'aspect trop différents. L'équation pour la fraction volumique 


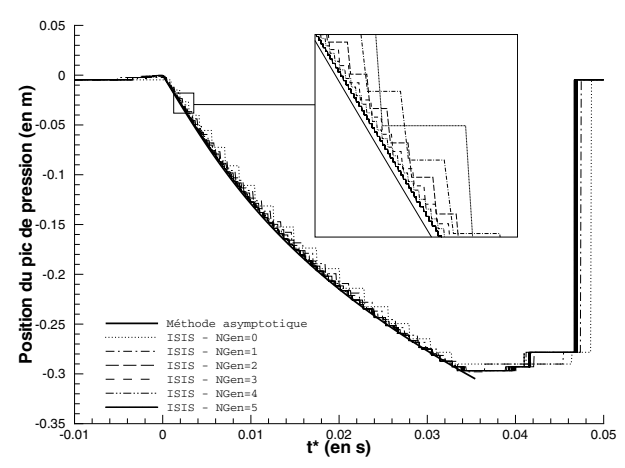

(a) Cas SymLight

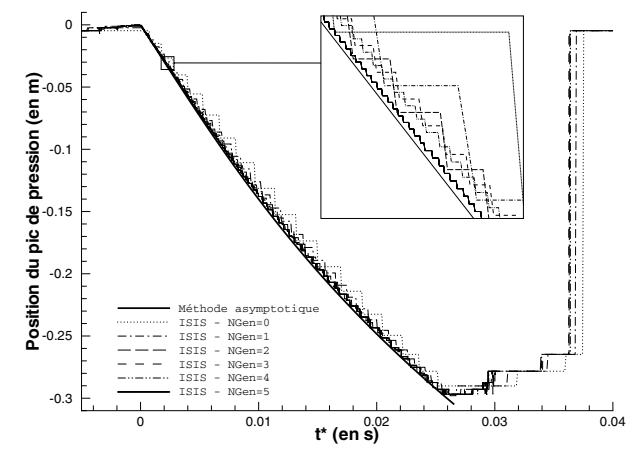

(b) Cas SymMedium

Fig. 12. Évolution temporelle de la position du pic de pression : cas symétrique.

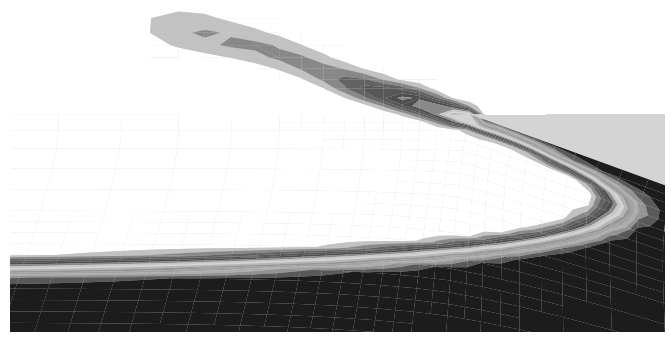

(a) $\mathrm{NGen}=1$

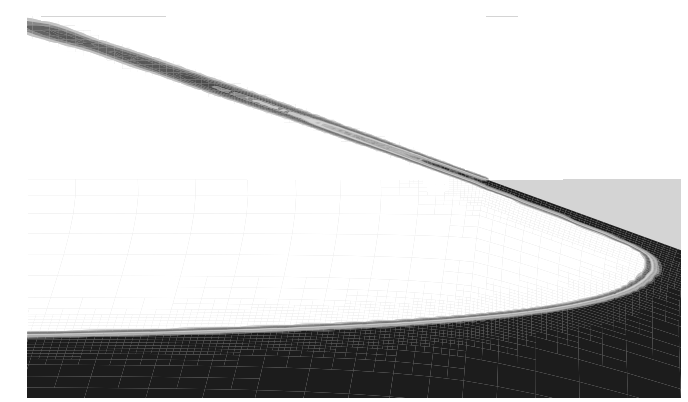

(b) $\mathrm{NGen}=3$

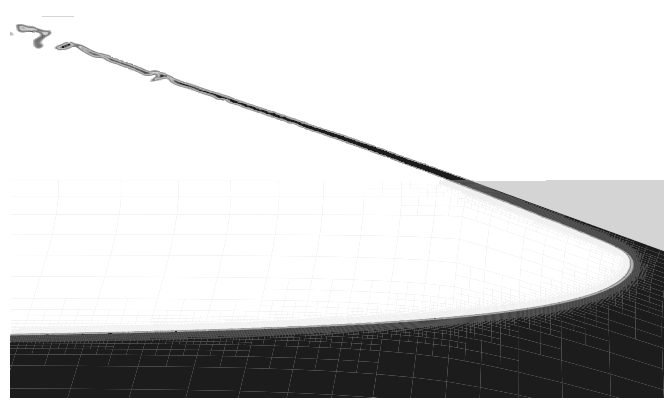

(c) $\mathrm{NGen}=5$

Fig. 13. Influence de NGen sur la définition de la surface libre.

est résolue à partir du schéma IGDS [4]. On est alors confronté à une contrainte sur le pas de temps pour assurer des nombres de courant Co faibles. Comme il est difficile de formuler a priori une loi analytique de $\delta t$ au cours des itérations temporelles qui satisfasse cette contrainte sur l'ensemble de la simulation sans aboutir à des pas de temps excessivement petits en de nombreux instants, une loi de pas de temps adaptative a été employée. En ce qui concerne le mouvement, les translations sont reprises par repositionnement en bloc, tandis que la rotation (pour les cas asymétriques) est gérée par pondération analytique.

Il apparaît sur l'ensemble des simulations effectuées une bonne représentation de la cinématique du prisme (et donc des efforts), même sur les maillages non adaptés (Fig. 10) : sur ces figures, la simulation « classique » sans raffinement a été effectuée avec un autre maillage disposant d'un nombre de points sensiblement équivalent à celui obtenu avec $\mathrm{NGen}=3$. On notera cependant que les calculs sont par nature dans l'impossibilité de capter les oscillations rapides engendrées par les vibrations de la structure, puisque celle-ci est modélisée par un corps indéformable. Bien que plus précise, l'utilisation de la procédure adaptative n'est donc pas fondamentale pour modéliser ce phénomène puisqu'il est déjà bien simulé à partir d'une grille non adaptée malgré la violence de la décélération du prisme qui est soumis à environ $10 \mathrm{~g}$, pour les cas présentés, dans les fractions de seconde qui suivent l'impact. Par contre, si l'on s'intéresse au pic de pression, l'intérêt de la procédure adaptative apparaît plus nettement : la capture de ce phénomène local et violent est rendue possible par l'emploi de celle-ci. Le processus adaptatif permet d'atteindre des solutions qui convergent en maillage; lorsque NGen croît, les courbes s'accumulent sur la solution asymptotique. Pour NGen $=5$, la solution est quasiment convergée et très proche d'une méthode asymptotique basée sur la théorie de Wagner [8], comme le montre la figure 11. La capture de ce phénomène par résolution des équations de Navier-Stokes est alors obtenue pour un nombre de points et un temps de calcul qui reste raisonnable. L'évolution de la position de ce pic de pression est aussi très bien capturée, comme on peut le voir sur la figure 12 .

Sur la figure 13, on retrouve aussi que plus le niveau de raffinement est élevé, plus la surface libre est capturée précisément (la diffusion de l'interface devenant plus faible). La figure 14 montre l'évolution de la surface libre à différents instants pour le cas AsymLight avec un nombre de générations de 3 . Le raffinement local permet là encore de capturer de manière fine la surface libre, tout 

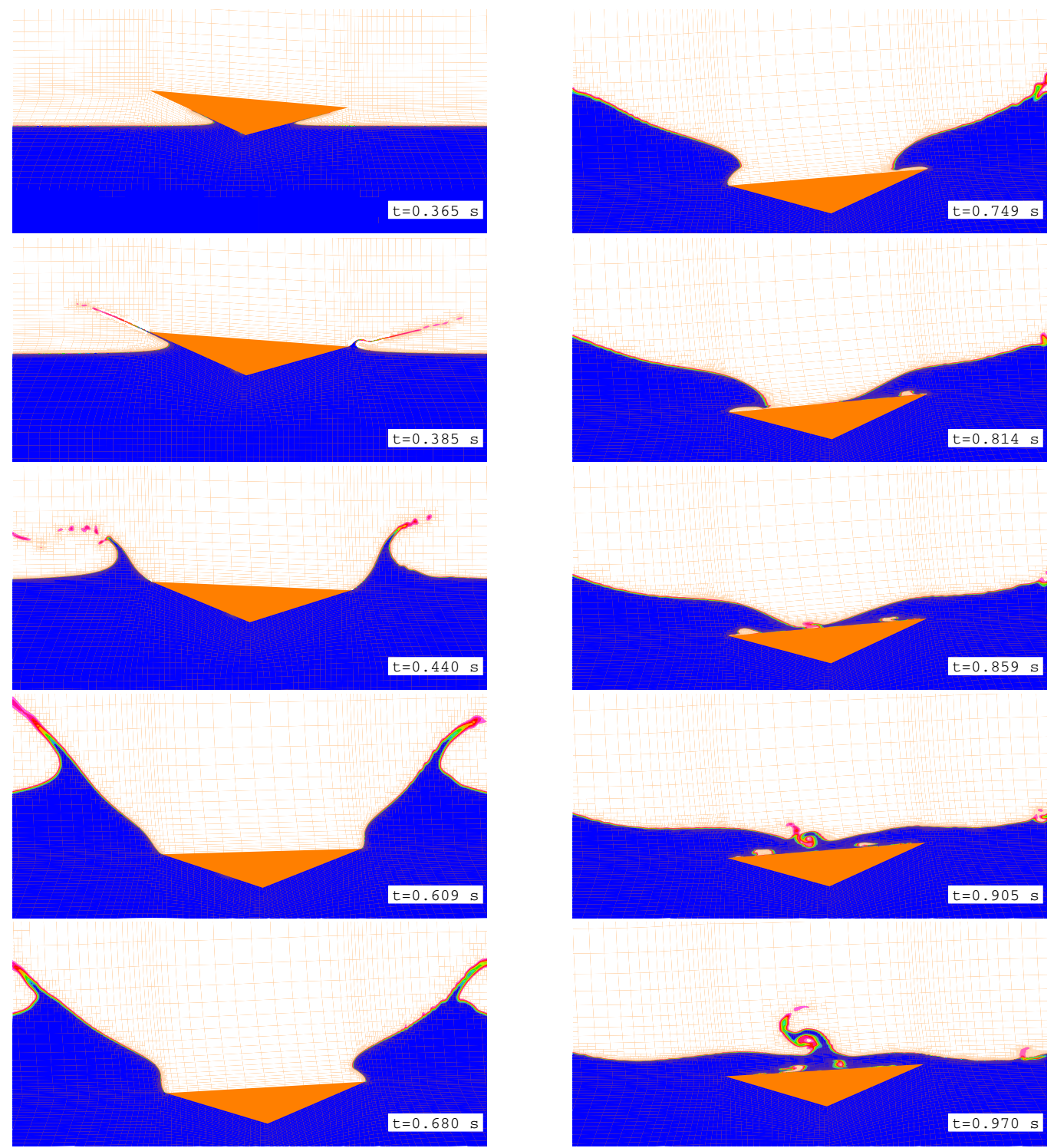

Fig. 14. Evolution de la surface libre : cas AsymLight.

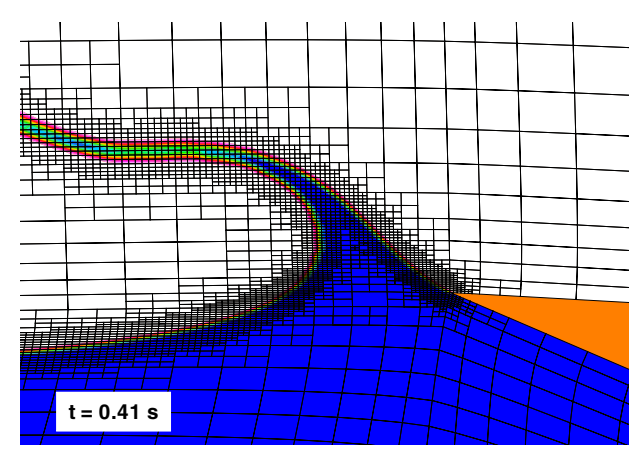

(a)

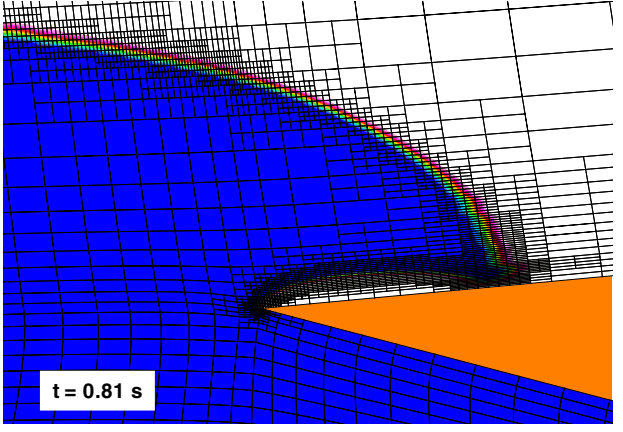

(b)

Fig. 15. Visualisation du raffinement de maillage : NGen $=3$. 
au long du calcul, même lorsque l'eau vient déferler sur la partie supérieure du corps (Figs. 14 et 15).

\section{Conclusions}

L'objectif de cet article était de présenter les méthodes numériques développées dans un solveur Navier-Stokes liées à l'interaction écoulement-mouvement et de montrer l'intérêt d'une utilisation conjointe avec des techniques d'adaptation de maillage. En effet, l'application liée à la simulation d'impacts a permis de mettre en exergue l'intérêt de l'association mouvement de corps/adaptation locale de maillage. Celui-ci s'est particulièrement révélé pour la capture du pic de pression. Cette quantité locale (mais sans pour autant être négligeable car dimensionnante) est difficilement accessible à partir de calculs mono-grilles. Par ailleurs, les calculs adaptatifs permettent d'assurer une description fine de la surface libre pendant et après l'impact pour un nombre de points et un temps de calcul qui restent raisonnables. La prochaine étape est de montrer la faisabilité de ce genre de calculs sur des configurations tridimensionnelles. Pour atteindre des niveaux de raffinement élevés, on ne pourra pas se passer d'un développement technique pour paralléliser les procédures adaptatives et traiter le problème de « load-balancing». En effet, si le raffinement s'effectue majoritairement sur un bloc donné, l'efficacité de l'ensemble des processus parallèles sera alors amoindrie puisque la vitesse du calcul sera limitée par le bloc contenant le plus grand nombre de cellules.

Ces simulations mettant en jeu des corps en mouvement ouvrent des champs d'application plus vastes et diversifiés : tenue à la mer, manœuvrabilité, biohydrodynamique, ... Concernant ce dernier point, elles sont d'ores et déjà exploitées dans le cadre du projet ROBEA [9], dont l'objectif est de concevoir un robot-anguille autopropulsé à locomotion anguilliforme. Pour cela, des simulations mettant en jeu un corps déformable à déformation imposée (mais dont le mouvement est résolu) ont été réalisées afin de valider un modèle de contact fluidestructure nécessaire à la commande du robot $[3,10]$.

Le cadre des corps déformables à déformation imposée pose bien entendu les bases d'une interaction fluidestructure complète dans laquelle la forme du corps ne serait plus imposée mais calculée en fonction des efforts s'exerçant sur celui-ci. Ce dernier point apparaît donc comme une suite logique. D'ailleurs des travaux sont actuellement en cours pour mettre en place une interaction fluide/structure pour des corps élancés de type poutre.

\section{Références}

[1] S. Patankar, D. Spalding, A calculation procedure for heat, mass and momentum transfer in three-dimensional parabolic flows, Int. J. Heat Mass Transfer 15 (1972) 1787-1806

[2] G. Deng, E. Guilmineau, P. Queutey, M. Visonneau, Capture et suivi d'interfaces d'écoulements de fluides visqueux incompressibles non-miscibles, in $8^{\text {e }}$ Journées de l'Hydrodynamique, Nantes, France, mars 2001, pp. 17-30

[3] A. Leroyer, M. Visonneau, Simulation d'un robotanguille par résolution des équations de Navier-Stokes, Mécaniques \& Industries 7 (2006) 189-199

[4] A. Leroyer, Étude du couplage écoulement/mouvement pour des corps solides ou à déformation imposée par résolution des équations de Navier-Stokes, Contribution à la modélisation numérique de la cavitation, Thèse, École Centrale de Nantes, France, 2004, disponible en ligne

ftp://ftp.ec-nantes.fr/pub/DMN/Thesis/ these_leroyer.pdf

[5] A. Hay, Étude des stratégies d'estimation d'erreur numérique et d'adaptation locale de maillages nonstructurés pour les équations de Navier-Stokes en moyenne de Reynolds, Thèse, École Centrale de Nantes, France, 2004, disponible en ligne

ftp://ftp.ec-nantes.fr/pub/DMN/Thesis/ these_hay.ps.gz

[6] A. Hay, M. Visonneau, Computation of free-surface flows with local mesh adaptation, Int. J. Numerical Methods in Fluids 49 (2005) 785-816

[7] L. Xu, A. Troesch, R. Peterson, Asymmetric hydrodynamic impact and dynamic response of a vessels, J. Offshore Mechanics and Arctic Engineering 121 (1999) 83-89

[8] Y.-M. Scolan, E. Coche, T. Coudray, E. Fontaine, Étude analytique et numérique de l'impact hydrodynamique sur des carènes dissymétriques, in : $7^{\mathrm{e}}$ Journées de l'Hydrodynamique, Marseille, France, mars 1999

[9] F. Boyer, M. Alamir, D. Chablat, W. Khalil, A. Leroyer, P. Lemoine, Robot anguille sous-marin en 3D, Techniques de l'Ingénieur 7 (2006) 856 1-32

[10] A. Leroyer, M. Visonneau, Numerical methods for RANSE simulations of a self-propelled fish-like body, J. Fluids and Structures 20 (2005) 975-991 